\title{
Saúde bucal no Brasil: análise do ciclo da política*
}

\author{
Oral Health in Brazil: Analysis of the Policy Cycle
}

Salud bucal en Brasil: análisis del ciclo de la política

Fecha de recepción: 11-09-2018 | Fecha de aceptación: 11-02-2019

\begin{abstract}
JOSÉ EUdES DE LORENA SOBRINHO
Universidade de Pernambuco, Recife, Pernambuco, Brasil. eudeslorena@ hotmail.com.

https://orcid.org/0000-0001-7820-735X
\end{abstract}

\begin{abstract}
Petrônio José de Lima Martelli
Universidade Federal de Pernambuco, Recife, Pernambuco, Brasil. petronio.martelli@ufpe.br. https://orcid.org/000-0001-6920-6435
\end{abstract}

* Investigación original

Correspondencia: eudeslorena@hotmail.com; petronio.martelli@ufpe.br

doi: https://doi.org/10.11144/Javeriana.uo38-80.sbba 
Como citar: Sobrinho JE, Martelli PJ. Saúde bucal no Brasil: análise do ciclo da política. Univ Odontol. 2019 ene-jun; 38(80). https://doi.org/10.11144/Javeriana.uo38-80.sbba

\section{RESUMO}

Antecedentes: A política nacional de saúde bucal do Brasil, instituída em 2004, é considerada a maior política pública deste segmento em todo o mundo. Objetivo: analisar a Política Nacional de Saúde Bucal do Brasil sob a teoria do ciclo das políticas públicas. Métodos: tratou-se de um estudo qualitativo baseado no policy cicle, que buscou analisar o ciclo da Política Nacional de Saúde Bucal do Brasil. Foram utilizados dois métodos para a coleta dos dados: a análise documental e a realização de entrevistas semiestruturadas. Resultados: embora há muito tempo se reconheçam os problemas de saúde bucal da população brasileira, foi no ano de 2003 que se abriu uma janela de oportunidades para que este tema ocupasse a agenda de prioridades do Governo Federal. Instituída em 2004, a política avançou em cobertura de serviços básicos e especializados no território nacional, atendendo a populações prioritárias. A partir do ano de 2016, no entanto, percebe-se sua estagnação dada a conjuntura político-econômica brasileira. Conclusões: os resultados das intervenções demonstram ter ocorrido melhora considerável nos indicadores de saúde bucal; e aumento da cobertura populacional atendida pelas ações e serviços de saúde bucal, fatos estes que recomendam a continuidade da política.

\section{Palavras-chave}

Brasil; ciclo da política; conjuntura político-econômica; cobertura populacional 


\section{Áreas temáticas}

atenção primária à saúde; odontologia; políticas públicas; política de saúde; saúde bucal; serviços de saúde bucal

\section{ABSTRACT}

Background: Brazil's national oral health policy, established in 2004, is considered the largest public policy in this segment worldwide. Objective: To analyze the National Oral Health Policy of Brazil under the theory of the cycle of public policies. Methods: This was a qualitative study based on the policy cycle, which sought to analyze the cycle of the Brazilian National Oral Health Policy. Two methods were used to collect the data: the documentary analysis and the performance of semi-structured interviews. Results: Although the oral health problems of the Brazilian population have long been recognized, it was in 2003 that a window of opportunity was opened for this topic to occupy the agenda of priorities of the Federal Government. Established in 2004, the policy has advanced in coverage of basic and specialized services in the national territory, serving priority populations. From the year 2016, however, one can perceive its stagnation given the Brazilian political-economic conjuncture. Conclusions: The results of the interventions show a considerable improvement in the oral health indicators; and an increase in the population coverage served by oral health services and services, facts that recommend the continuity of the policy.

\section{Keywords}

Brazil; policy cycle; political-economic conjuncture; population coverage 


\section{Thematic fields}

dental health services; dentistry; health policy; oral health; primary health care; public policy

\section{RESUMEN}

Antecedentes: La política nacional de salud bucal de Brasil, instituida en 2004, es considerada la mayor política pública de este segmento en todo el mundo. Objetivo: analizar la Política Nacional de Salud Bucal de Brasil bajo la teoría del ciclo de las políticas públicas. Métodos: se trató de un estudio cualitativo basado en el policy cicle, que buscó analizar el ciclo de la Política Nacional de Salud Bucal de Brasil. Se utilizaron dos métodos para la recolección de los datos: el análisis documental y la realización de entrevistas semiestructuradas. Resultados: aunque desde hace mucho tiempo se reconocen los problemas de salud bucal de la población brasileña, fue en el año 2003 que se abrió una ventana de oportunidades para que este tema ocupara la agenda de prioridades del Gobierno Federal. Instituida en 2004, la política avanzó en cobertura de servicios básicos y especializados en el territorio nacional, atendiendo a las poblaciones prioritarias. A partir del año 2016, sin embargo, se percibe su estancamiento dada la coyuntura política-económica brasileña. Conclusiones: los resultados de las intervenciones demuestran que se ha producido una mejora considerable en los indicadores de salud bucal; y aumento de la cobertura poblacional atendida por las acciones y servicios de salud bucal, hechos que recomiendan la continuidad de la política.

\section{Palabras clave}


Brasil; ciclo de la política; coyuntura político-económica; cobertura de la población

\section{Áreas temáticas}

atención primaria de salud; odontología; política de salud; política pública; salud bucal; servicios de salud oral

\section{INTRODUÇÃO}

As políticas públicas são resultantes da atividade política e compreendem o conjunto das decisões e ações relativas à alocação dos recursos públicos (1). Caracterizada como uma ação multidisciplinar que visa interpretar as causas e consequências da ação do Estado, a análise de políticas públicas passou a ser uma linha de pesquisa utilizada com maior frequência e aceitabilidade pelos próprios governos a partir da década de setenta, embora as ciências sociais já tivessem interesse neste campo há algum tempo (2).

O instrumental de análise de política diferencia três dimensões da palavra política: "polity" se referindo às instituições políticas e ao sistema político; "politics" como uma dimensão do processo político. E "policy" como uma dimensão material, referindo-se às políticas públicas em si (3). Assim, os estudos podem ser agrupados em: "analysis of policy" e "analysis for policy". A primeira, mais descritiva, tem como objetivo produzir conhecimentos sobre o processo de elaboração política. A segunda, de caráter prescritivo, busca auxiliar os formuladores de política, agregando conhecimento ao processo de elaboração de políticas, envolvendo-se diretamente na tomada de decisões (4-5). 
Utilizar de modelos teóricos para dar conta deste emaranhado complexo é uma exigência para os estudiosos em análise das políticas públicas (1), sendo o "ciclo das políticas públicas” um dos mais empregados, o qual corresponde a um esquema de visualização e interpretação que organiza a vida de uma política pública em fases sequenciais e interdependentes (6).

Diversas versões foram desenvolvidas para a sua visualização. Neste trabalho, optou-se pelo modelo composto por sete fases principais denominadas de identificação do problema, formação da agenda, formulação de alternativas, tomada de decisão, implementação, avaliação e extinção (7), que pode ser visualizado na (Figura 1).

Organizadas logicamente na perspectiva acadêmica, elas se apresentam misturadas e alternadas na vida prática. Isto porque não há pontos de início e finalização de uma política pública e as fronteiras entre as fases não são nítidas, sendo o seu processo incerto (8).

FIGURA 1

CiClO DAS POLÍTICAS PÚBLICAS. EXTRAÍDO DE SECCHI (7)

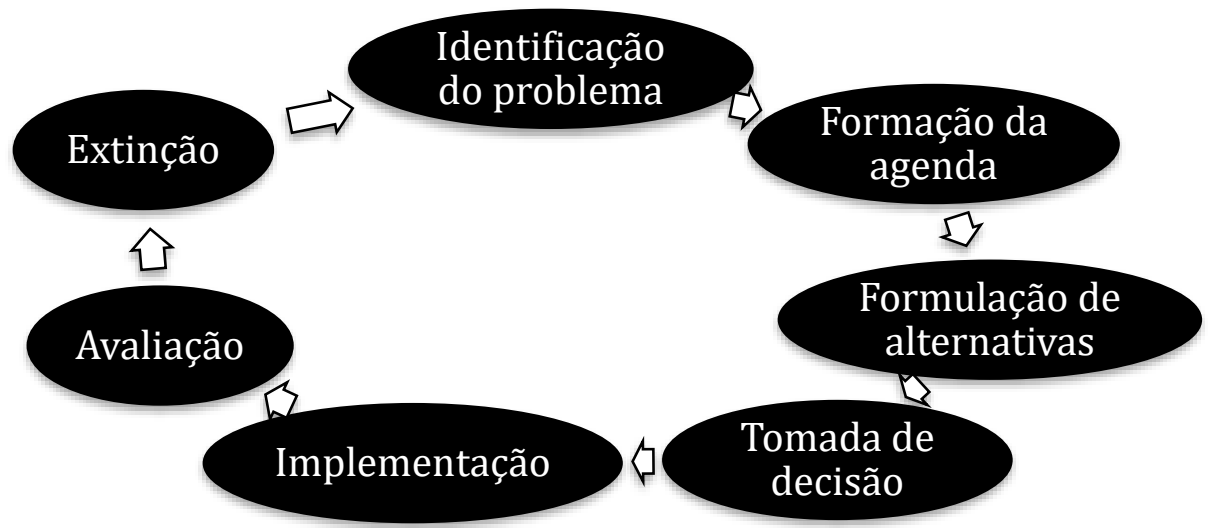


Estudos sobre o ciclo da política tem sido cada vez mais utilizados na América Latina, mas não específicos para a saúde bucal, dada a sua incipiente inserção nos sistemas de saúde. Particularmente, será estudado o cenário brasileiro da implementação da política denominada "Brasil Sorridente", buscando-se responder à seguinte pergunta: Como a Política Nacional de Saúde Bucal foi implantada e está sendo implementada no Brasil? Deste modo, este artigo objetiva analisar a Política Nacional de Saúde Bucal do Brasil (PNSB) sob a teoria do ciclo das políticas públicas.

\section{MATERIAIS E MÉTODOS}

Tratou-se de um estudo qualitativo baseado no policy cicle (6) que buscou analisar o ciclo da PNSB. Foram utilizados dois métodos para a coleta dos dados: a análise documental e a realização de entrevistas semiestruturadas.

A análise documental consiste em operações que visam estudar e analisar documentos para descobrir as circunstâncias sociais e econômicas com as quais podem estar relacionados (9). Foram seguidas as fases propostas por Bardin: pré-análise, análise do material e tratamento dos resultados (10). Incluíram-se documentos oficiais publicados pelo Ministério da Saúde, Conselho Nacional dos Secretários Estaduais de Saúde (Conass), Conselho Nacional dos Secretários Municipais de Saúde (Conasems), Conselho Federal de Odontologia (CFO), Conselho Nacional de Saúde (CNS), entre outros.

Em seguida, foram realizadas entrevistas com oito atores estratégicos envolvidos na formulação, 
implantação e implementação da política nacional de saúde bucal do Brasil, sendo três profissionais da Coordenação Nacional de Saúde Bucal do Ministério da Saúde atuantes no período 2004-2013, dois membros da Comissão de Assessoramento à Coordenação Nacional de Saúde Bucal que atuaram no período 2004-2008, um membro do Conselho Nacional de Saúde no período 2005-2006, um membro do Conselho Federal de Odontologia e um membro do Departamento de Atenção Básica do Ministério da Saúde do período 2004-2013.

A presente pesquisa foi aprovada pelo Comitê de Ética em Pesquisa (CEP) do Centro de Pesquisas Aggeu Magalhães/Fundação Oswaldo Cruz (CAAE 31601814.2.0000.5190, Parecer 724.869). Todas as etapas da pesquisa foram realizadas por um único pesquisador e, para garantir o anonimato dos entrevistados, estes foram identificados com letras do alfabeto.

\section{RESULTADOS}

Nesta seção, serão apresentados os resultados agregando os dados quantitativos e qualitativos coletados, nas sete etapas que constituem o ciclo da política, referencial teórico adotado nesta pesquisa:

1. Identificação do Problema: com a inexistência de uma política pública na área, eram alarmantes os indicadores de saúde bucal da população brasileira, sendo este o problema identificado para a sequência de etapas do ciclo da política.

2. Formação da agenda: embora em diferentes momentos da história da saúde pública brasileira, sobretudo a partir do ano da criação do Sistema Único de Saúde (1988), iniciativas pontuais de municípios e estados ofereciam assistência odontológica de baixa complexidade sem, contudo, 
incluir esta área na agenda de prioridades do Governo Federal. Com a eleição do Presidente Lula, em 2002, abrem-se janelas de oportunidade para a formulação da PNSB.

3. Formulação de alternativas: foram formuladas as Diretrizes da PNSB em 2003, em discussão que envolveu diferentes atores políticos e entidades governamentais e não-governamentais.

4. Tomada de decisão: no ano de 2004 foi implantada a PNSB Brasil Sorridente.

5. Implementação: houve ampliação do quantitativo das Equipes de Saúde Bucal (ESB) na Atenção Básica, implantação dos Centros de Especialidades Odontológicas (CEO) e Laboratórios Regionais de Prótese Dentária (LRPD) na Média Complexidade; e ofertados procedimentos odontológicos na alta complexidade.

6. Avaliação: diferentes mecanismos foram implementados para monitorar e avaliar a PNSB em seus anos de funcionamento, a exemplo do Programa Nacional de Melhoria do Acesso e da Qualidade da Atenção Básica (PMAQ-AB) e dos CEO (PMAQ-CEO); e realizadas pesquisas nacionais de saúde bucal para estimar a prevalência dos principais agravos na população brasileira, anos de 2003 e 2010.

7. Extinção: com os resultados obtidos em termos de cobertura de serviços, número de procedimentos odontológicos e impactos na redução da prevalência da cárie dentária e outros agravos em saúde bucal, a PNSB permanece em vigor. Entretanto, o cenário após o afastamento da Presidenta Dilma, em 2016, trouxe riscos à ampliação desta Política.

\section{DISCUSSÃO}

O histórico da assistência odontológica no Brasil construído sobre a odontologia liberal e da odontologia previdenciária, e mais recentemente ampliada pela odontologia pública e odontologia 
suplementar, ajudam a entender o conflito de forças ideológico-políticas divergentes dos grupos de profissionais e técnicos dos setores (11).

Os segmentos interessados na realização das políticas públicas de saúde bucal não ocuparam os postos e os cargos de poder durante a década de 90 , permanecendo em tais funções as elites que representavam os interesses privados no interior da esfera pública; o que decorreu em modelos assistenciais de saúde bucal pouco apropriados à realidade epidemiológica (12).

Foi na segunda metade da década de setenta que a "luta pela formulação e implementação de uma política pública de saúde bucal" passou a ocupar as agendas dos movimentos sociais de estudantes de Odontologia, embora programas verticalizados tivessem sido implementados nacionalmente, a exemplo do Programa de Prevenção da Cárie e do Câncer de Boca e a tentativa de fluoretação das águas de abastecimento público pelo Serviço Especial de Saúde Pública (SESP). (13).

Tais circunstâncias são corroboradas pelo relato do entrevistado A: o processo de construção da PNSB remonta à época do Movimento da Reforma Sanitária Brasileira quando já havia movimentos em prol da inserção da Odontologia nas discussões. A saúde bucal foi tratada em diversas Conferências Nacionais de Saúde.

Em 1986 foi realizada a $1^{\text {a }}$ Conferência Nacional de Saúde Bucal como desdobramento da $8^{\text {a }}$ Conferência Nacional de Saúde. Os temas abordados foram: saúde como direito de todos e dever do estado; diagnóstico de saúde bucal no Brasil; reforma sanitária: inserção da Odontologia no Sistema Único de Saúde; financiamento do setor de saúde bucal (14). Neste mesmo ano foi criada 
a Divisão Nacional de Saúde Bucal no Ministério da Saúde. A ela caberiam, dentre outras coisas, fornecer subsídios para a definição da Política Nacional de Saúde Bucal (15).

Mesmo com inúmeras limitações estruturais e oposição politico-ideológica, a Divisão conseguiu concluir o "Levantamento Epidemiológico em Saúde Bucal: Brasil, zona urbana, 1986" (12). Durante o Governo Collor (1990-1991), o novo diretor da Divisão Nacional de Saúde Bucal se dedicou ao Programa Nacional de Controle da Cárie pelo Método de Fluoretação do Sal, sem grandes repercussões. Neste período, a Divisão é rebaixada ao nível de Coordenação e perde força política (12). Os programas nacionais de saúde bucal lançados foram extintos e nenhum documento norteou a política de saúde bucal no âmbito federal (16).

Em 1993 foi realizada a 2 ${ }^{\text {a }}$ Conferência Nacional de Saúde Bucal, onde se destacou que a efetiva inserção da Saúde Bucal no Sistema Único de Saúde se daria mediante um processo sob controle da sociedade. No governo Itamar Franco (1993-1994) houve expressivo declínio das ações de saúde bucal no Ministério da Saúde, com baixíssima prioridade para a Política Nacional de Saúde Bucal (15).

A saúde bucal não ocupou espaço mesmo com a institucionalização do Programa Saúde da Família (PSF) em 1994, com caráter substitutivo ao modelo de atenção básica tradicional, orientado pelos princípios de adscrição de clientela, territorialização, diagnóstico da situação de saúde da população e planejamento baseado na realidade local (17).

Novo Levantamento Epidemiológico Nacional de Saúde Bucal fora realizado no ano de 1996 em 
crianças entre 6 e 12 anos de idade das 26 capitais e do Distrito Federal. O indicador CPO-D (dentes cariados, perdidos e obturados) apresentou media de 0,28 aos 06 anos de idade e 3,06 aos $12 \operatorname{anos}(18)$.

Apesar dos avanços percebidos no setor da atenção básica na década de noventa, muito pouco se fez no âmbito específico da saúde bucal. O Governo Federal desconsiderou as propostas das Conferências Nacionais de Saúde Bucal, das Conferências Nacionais de Saúde e dos demais espaços de participação popular, como fruto de uma política neoliberal (14). Iniciativas locais de assistência em saúde bucal no Sistema Único de Saúde aconteceram em alguns municípios como São Paulo, Santos, Porto Alegre, Campinas, Belo Horizonte, Curitiba e Sobral (19).

$\mathrm{Na}$ análise dos conferencistas, a assistência em saúde bucal era insuficiente, restrita a poucos procedimentos, focalizada em grupos específicos e, diante desta situação, propuseram a implementação de um programa de saúde bucal no SUS (20). O Governo Federal iniciou neste ano o projeto SB Brasil 2000 - Pesquisa Nacional de Saúde Bucal, que apenas seria concluída no Governo Lula.

Ainda em dezembro de 2001, o Ministério da Saúde publicou a Portaria $\mathrm{n}^{\circ} 1.444$ que estabeleceu o incentivo financeiro para as ações e inserção dos profissionais de saúde bucal no PSF, com a configuração de dois tipos de Equipes de Saúde Bucal (ESB). Porém, uma ESB seria implantada a cada duas Equipes de Saúde da Família (21).

Nas eleições presidenciais do ano de 2002, o então candidato do Partido dos Trabalhadores 
apresentou o seu programa de governo da área da saúde denominado "Saúde para a família brasileira", no qual inseriu a saúde bucal como uma das suas prioridades, fato até então inédito em campanhas presidenciais. Rompendo com a hegemonia das ideias liberalistas que ocupavam os governos, até então.

Para um dos entrevistados neste estudo, tais proposições presentes no Programa de Governo do candidato Lula são entendidas como o início da construção do "Brasil Sorridente":

[...] a construção da Política Nacional de Saúde Bucal se iniciou desde as campanhas quando o Presidente Lula era candidato. Então isto foi se amadurecendo no início do Programa do Governo do Lula (entrevistado B).

Dentre os motivos que justificam a atenção conferida pelo candidato e, posteriormente, Presidente da República, à questão da saúde bucal, foram citados:

[...] primeiro um grupo de sanitaristas vinculado à saúde bucal que militava em órbita do Partido dos Trabalhadores, do Presidente Lula; e, segundo, de uma demanda pessoal do presidente, mesmo antes de ele ser presidente, por entender que a saúde bucal precisava se construir na área pública. Isto era percebido [...] por duas questões principais: primeiro a origem do presidente: é um representante das necessidades de saúde do povo brasileiro, e a saúde bucal evidentemente era uma delas; segundo pela sua militância no sindicato, porque lá também desaguava na questão dos trabalhadores a necessidade do atendimento a tratamento odontológico (entrevistado C).

Após a vitória do candidato Luiz Inácio Lula da Silva, militantes partidários se reuniram em São 
Paulo no dia 24 de novembro de 2002 para debater as propostas de uma Política Nacional de Saúde Bucal que considerasse os anseios históricos dos movimentos sociais e sanitários. Foi formulado um documento intitulado "Fome Zero e Boca cheia de dentes!"

Para o entrevistado D, este documento fora de extrema importância, pois: no início do Governo Lula quando foi lançado o documento "Fome Zero e Boca Cheia (de dentes)!" apontava pro então Humberto Costa, o Lula ainda não tinha tomado posse, logo mais ao futuro ministro Humberto Costa, a necessidade de nós colocarmos em curso uma política de forma orgânica ao Sistema Único de Saúde que contemplasse os diversos níveis de atenção (atenção primária, secundária e terciária). Abriu-se aí uma janela de oportunidades, no sentido de convencer a equipe de transição sobre a necessidade de um projeto político na área de saúde bucal dentro da agenda de prioridades do governo (22). Somam-se ao momento politico favorável alguns outros argumentos:

[...] A Política de Atenção Primária já em consolidação, os PSF no Brasil eles tinham uma estrutura ramificada com tradição forte de Atenção Primária em Saúde. Então isto foi em dada medida um facilitador [para a PNSB]. Existia de fato uma demanda política, uma demanda societária, que diziam: essas pessoas clamam, essas pessoas precisam ser atendidas de algum jeito (entrevistado A).

Interessante que o SB, o levantamento de saúde bucal que todos nós participamos no ano 2000 me parece, também ele foi importante como um indicador da necessidade do povo brasileiro por uma política de saúde bucal que todos os governos anteriores não priorizaram (entrevistado B). 
A grande demanda reprimida que se colocava; e uma percepção do Presidente da necessidade de construir a saúde dentro de uma perspectiva de integralidade e vendo a saúde bucal neste contexto (entrevistado C).

Algumas ações realizadas no primeiro ano do Governo Lula já mostram as tendências que seriam observadas: a conclusão da Pesquisa Nacional de Saúde Bucal SB Brasil iniciada no ano 2000; a convocação da $3^{\text {a }}$ Conferência Nacional de Saúde Bucal; a definição das linhas de atuação do governo federal para a área; e a área técnica de Saúde Bucal se tornou novamente Coordenação Nacional de Saúde Bucal (16).

Passou-se então ao momento de formulação daquela que viria a ser a nova Política Nacional de Saúde Bucal do Brasil com a consequente tomada de decisão por sua implementação no país.

Uma Comissão de Assessoramento à Coordenação Nacional de Saúde Bucal foi formalizada por meio de Portaria $\mathrm{n}^{0} 36$ de 14 de janeiro de 2004, sendo composta por Cleber Ronald Inácio dos Santos, Christian Mendez Alcântara, Helenita Corrêa Ely, Idiana Luvison, José Carrijo Brom, Marco Aurélio Peres, Marco Manfredini, Marcos Werneck, Mauro Rubem de Menezes Jonas, Moacir Tavares Martins Filho, Paulo Capel Narvai, Petrônio Martelli e Rosângela Camapum (23). A Comissão de Assessoramento funcionou durante os dois Governos Lula. Na verdade, o que a Comissão fez foi sintetizar o que era produzido nos eventos, ou seja, nas reuniões, nos eventos de institucionalização da PNSB. Ela sistematizou o que tinha sido acordado nas Conferências de Saúde Bucal e, em dada medida, sistematizou o que era apontado nos Encontros Nacionais dos Técnicos do Serviço Público Odontológico (ENATESPO), e 
também já com os Congressos de Saúde Bucal Coletiva que ocorriam sempre junto ao ENATESPO. Eram fóruns que tratavam de debate e apontavam diretrizes (entrevistado C).

Oriundo das discussões desta Comissão um documento preliminar foi apresentado e analisado pelas Coordenações Estaduais de Saúde Bucal e após sugestões elaboradas por estas Coordenações, bem como apresentação a todas as entidades odontológicas, o documento recebeu a versão final em dezembro de 2003 (24). Tais meios de elaboração da PNSB permitem afirmar que:

Esta construção ao longo do tempo foi coletiva, porque um conjunto de estudiosos, pesquisadores, trabalhadores, fez com que ela tenha chegado a este estágio atual que a gente tem (entrevistado E).

Em fevereiro de 2004, o próprio coordenador nacional de saúde bucal, Gilberto Pucca, apresentou na 139ª Reunião do Conselho Nacional de Saúde a proposta do Ministério da Saúde das diretrizes da Política Nacional de Saúde Bucal. Durante esta reunião foram apresentadas a situação de saúde bucal da população brasileira com base no SB Brasil 2003, os resultados das ações desenvolvidas no primeiro ano do Governo Lula e as propostas da PNSB. Após aprovação pelo Conselho Nacional de Saúde, estas diretrizes foram aprovadas em reunião da Comissão Intergestores Tripartite ocorrida também em fevereiro de 2004.

[...] o Brasil Sorridente passou por todas as instâncias da participação popular do ponto de vista oficial e culminando com a aprovação na Comissão Tripartite antes do lançamento. Ele foi construído dentro desta articulação institucional e controle social (entrevistado F). 
Em 17 março de 2004, na cidade de Sobral, Ceará, foi lançado o Programa Nacional de Saúde Bucal "Brasil Sorridente", numa clara tomada de decisão pela execução da PNSB por parte do Presidente Lula. Alguns trechos de seu discurso revelam as justificativas para o seu posicionamento:

[...] desde a campanha de 1989 eu tive a primeira briga, enquanto candidato, por conta da saúde bucal no Brasil [...] E por que a saúde bucal nunca foi tratada como uma questão de saúde? Porque a questão da saúde bucal é uma doença de pobre, não é uma doença de rico. Rico começa a tratar o dente ainda quando tem dente-de-leite. Pobre só começa a tratar o dente quando os dentes começam a cair ou a doer demais. Então, por ser uma doença de pobre, o tratamento odontológico nunca foi colocado nos grandes planos de saúde como uma coisa necessária e importante para o nosso país (25).

O que está sendo feito aqui [...] é uma coisa que vai passar para a História. Levar a sério a saúde bucal do nosso povo porque a boca $[. .$.$] nunca foi tratada com o menor respeito por todos aqueles$ que pensam a saúde neste país (25).

Em consonância com o seu discurso e com as prioridades de governo estabelecidas, o tema da $3^{\text {a }}$ Conferência Nacional de Saúde Bucal, ocorrida entre os dias 29 de julho e $1^{\circ}$ de agosto de 2004, foi “Acesso e Qualidade Superando a Exclusão Social”. O debate ocorreu a partir de quatro eixos temáticos: 1) Educação e Construção da Cidadania; 2) Controle Social, Gestão Participativa e Saúde Bucal; 3) Formação e Trabalho em Saúde Bucal; e 4) Financiamento e Organização da Atenção em Saúde Bucal (26). 
Ressalte-se que a $3^{\text {a }}$ Conferência Nacional de Saúde Bucal fora precedida por 2.542 Conferências

Municipais ou Regionais de Saúde Bucal, 26 etapas estaduais e uma etapa Distrital; e teve papel

fundamental na avaliação das Diretrizes da PNSB (14).

Por fim, a saúde bucal foi inserida entre as iniciativas prioritárias elencadas pelo Ministério da Saúde no Plano Plurianual 2004-2007 “Um Pacto pela Saúde no Brasil”, aprovado pela Portaria $\mathrm{n}^{\mathrm{o}} 2.607$ de 10 de dezembro de 2004 .

O processo de implementação da PNSB se deu com publicações de portarias e outros dispositivos legais que autorizaram novos serviços, incluíram procedimentos odontológicos e ações similares (Tabelas 1 e 2).

TABELA 1

MARCOS HISTÓRICOS DA IMPLEMENTAÇÃO NO GOVERNO LULA GOVERNO LUIZ INÁCIO LULA DA SILVA ( $1^{\circ}$ de janeiro de 2003 a $1^{\circ}$ de janeiro de 2011)

\begin{tabular}{|c|c|}
\hline 2003 & $\begin{array}{l}\text { Conclusão do SB Brasil 2000; Portaria } n^{\circ} 673 \text { atualizou e reviu o incentivo financeiro às ações } \\
\text { de saúde bucal no PSF. }\end{array}$ \\
\hline 2004 & $\begin{array}{l}\text { Portaria } \mathrm{n}^{\circ} 36 \text { constituiu a Comissão de Assessoramento da Coordenação Nacional de Saúde } \\
\text { Bucal; } 3^{\mathrm{a}} \text { Conferência Nacional de Saúde Bucal; Formulação da PNSB "Brasil Sorridente"; } \\
\text { Implantação dos CEO e LRPD pela Portaria n }{ }^{\circ} \text { 1.570; Ampliação do financiamento das ESB pela } \\
\text { Portaria } \mathrm{n}^{\circ} 74 \text {.; Portaria } \mathrm{n}^{\circ} 1572 \text { definiu valores de custeio para próteses dentárias. }\end{array}$ \\
\hline 2005 & Emissão da AIH pelo cirurgião-dentista e criação da Política Nacional de Atenção Oncológica. \\
\hline 2006 & $\begin{array}{l}\text { Portaria } n^{\circ} 648 \text { instituiu a Política Nacional de Atenção Básica, definindo as responsabilidades e } \\
\text { atribuições da equipe de saúde bucal; Portaria } n^{\circ} 650 \text { revisa financiamento das ESB }\end{array}$ \\
\hline 2007 & $\begin{array}{l}\text { Decreto Presidencial no } 6.286 \text { de } 05 \text { de dezembro de } 2007 \text { instituiu o Programa Saúde na Escola, } \\
\text { incluindo ações de saúde bucal. }\end{array}$ \\
\hline 2008 & $\begin{array}{l}\text { Portaria } \mathrm{n}^{\circ} 90 \text { incluiu residentes em assentamentos da reforma agrária e remanescentes de } \\
\text { quilombos no cálculo do teto das equipes de saúde bucal; Divulgação do Caderno } 17 \text { de Saúde } \\
\text { Bucal; Portarias } n^{\circ} 2.489 \text { e } 3.066 \text { ampliam o financiamento das ESB. }\end{array}$ \\
\hline 2009 & $\begin{array}{l}\text { Instituído as Unidades Odontológpela Portaria } 2.371 \text { de } 07 \text { de outubro de } 2009 \text {; ampliação do } \\
\text { custeio das próteses dentárias pela Portaria } \mathrm{n}^{\circ} 2.372 \text {; Criado plano de fornecimento de } \\
\text { equipamentos odontológicos pela Portaria } \mathrm{n}^{\circ} 2.372 \text {. }\end{array}$ \\
\hline 2010 & $\begin{array}{l}\text { Portaria } \mathrm{n}^{\circ} 1.032 \text { incluiu procedimentos odontológicos para atendimento a pacientes com } \\
\text { necessidades especiais; Realização do SB Brasil } 2010 \text {. }\end{array}$ \\
\hline
\end{tabular}


TABELA 2

MARCOS HISTÓRICOS DA IMPLEMENTAÇ̃̃O NO GOVERNO DILMA

GOVERNO DILMA VANA ROUSSEFF

( $1^{\circ}$ de janeiro de 2011 a 31 de agosto de 2016)

12011 Criada a Atenção Domiciliar pela Portaria $\mathrm{n}^{\circ} 2.527$, incluindo a saúde bucal; PNAB foi revisada Portaria $\mathrm{n}^{\circ} 2.488$ criando ESF Ribeirinhas e Fluviais, e as Equipes de Consultório na Rua; Formuladas as Diretrizes do Componente Indígena da PNSB; Programa de Melhoria do Acesso e da Qualidade (PMAQ) no componente Atenção Básica contemplou a adesão das ESB; Portaria $\mathrm{n}^{\circ} 1.599$ amplia financiamento das ESB; Portaria $\mathrm{n}^{\mathrm{o}} 211$ amplia custeio das próteses dentárias; Lançamento do Plano Brasil Sem Miséria, incluindo a saúde bucal.

22012 Portaria $\mathrm{n}^{\circ} 1.341$ ampliou os valores da implantação e custeio mensal dos CEO; Portaria $\mathrm{n}^{\circ}$ 975 inclui os CEO na Rede de Cuidados à Pessoa com Deficiência do Ministério da Saúde; Portaria $\mathrm{n}^{\circ} 1.825$ amplia custeio para próteses dentárias

22013 Portaria $\mathrm{n}^{\circ} 261$ ampliou o PMAQ para os CEO; Portaria $\mathrm{n}^{\circ} 978$ ampliou financiamento das ESB.

Merecem ainda destaque, dentro da implementação da PNSB, a criação de uma rede de Centros Colaboradores (CECOL) credenciados pelo Ministério da Saúde para apoiar ações de vigilância da saúde bucal desenvolvidas no âmbito do Sistema Único de Saúde. A constituição da Rede CECOL foi proposta pelo Comitê Técnico Assessor (CTA) de Vigilância em Saúde Bucal, criado por meio da Portaria MS/SAS nº 939, de 21/12/2006. O CTA elaborou o plano de estruturação e implantação do componente de vigilância da saúde bucal da Política Nacional de Saúde Bucal e deu início, no final de 2007, à instalação de oito CECOL em universidades brasileiras (27).

A avaliação é o último estágio do ciclo da política, no qual se realiza a verificação de como a política está funcionando. Como resultado, uma política pode ser considerada bem sucedida e continuar no seu formato atual, pode ser julgada como necessitando de ajustes ou pode ser extinta, quando do seu insucesso (7).

É possível avaliar a PNSB sob duas dimensões: quanto às ações e serviços de saúde bucal e quanto à situação de saúde bucal da população brasileira. Os resultados alcançados são expressivos na atenção básica, pois em 2013 o país apresentou 38\% de cobertura populacional com serviços em 
quase 90\% dos municípios brasileiros. Um crescimento de 543\% na série histórica de 2002 a 2013. Eram 4.261 ESB em 2002, passando a 24.279 em 2014. Na atenção secundária, o número de Centro de Especialidades Odontológicas passou de 60 no ano de 2004 para 1.030 no ano de 2014; e quanto aos Laboratórios Regionais de Prótese Dentária também houve forte expansão, passando de 676 no ano de 2010 para 1.955 em 2014 (28)

O cenário a partir de 2015 se modificou: na atenção básica, reduziu-se ano após ano o número de ESB (de 24.467 em 2015, 24.383 em 2016 e 24.053 em 2017). Houve estagnação no número de CEO do país nos anos de 2015 e 2016, totalizando 1.034 unidades. Acompanhando a tendência, o número de LRDP em 2015 foi reduzido, para 1.770 (28). Efeitos das medidas econômicas para controle de gastos públicos podem então já ser observados na PNSB, tomando por pressuposto a agenda de austeridade fiscal implementada a partir do Governo Temer.

Quanto à situação de saúde bucal da população, um dos resultados mais importantes se refere ao fato de o país assumir a situação de país com baixa prevalência de cárie dentária, em virtude da redução do indicador CPO-D aos 12 anos de idade. Houve redução do CPO-D em todas as faixas etárias estudadas, tendo ocorrido aumento no percentual de indivíduos livres de cárie dentária em crianças, adolescentes e adultos e redução do número de dentes perdidos em crianças, adolescentes, adultos e idosos (29).

Foi identificado acréscimo nos percentuais de uso de prótese dentária inferior e superior em adolescentes e idosos, refletindo a situação de melhoria na cobertura de ações e serviços de reabilitação oral. Redução na necessidade de prótese dentária entre adolescentes foi constatada no 
período em virtude das ações de saúde bucal desenvolvidas prioritariamente para este público (29). Os argumentos expostos sustentam a boa avaliação da PNSB, sugerindo sua continuidade ao longo do tempo, com ajustes sendo realizados à medida que novas necessidades de saúde bucal venham a surgir.

Há, no entanto, dificuldades no presente e futuro da política nacional de saúde do Brasil que decerto ocasionarão novas demandas para a PNSB, tais como: a mudança na forma de repasse de recursos financeiros do Governo Federal para estados e municípios; a proposta de criação dos planos populares de saúde e a lei que congela os gastos públicos por vinte anos.

\section{CONCLUSÕES}

A análise da PNSB, dentro do ciclo da política, permite afirmar que o contexto político do país a partir do ano de 2003 favoreceu a sua formulação de forma ampla e participativa pela Comissão de Assessoramento à Coordenação Nacional de Saúde Bucal e pelas entidades de classe odontológica (CFO e Conselhos Regionais), garantindo inclusive o controle social por meio das instâncias colegiadas (Conselho Nacional de Saúde, Comissão Intergestores Tripartite).

O processo de implementação vem se constituindo ao longo do tempo, pautado no fortalecimento da saúde bucal na atenção básica e especializada, com foco na ampliação do acesso com qualidade, destacando-se o incentivo à implantação das Equipes de Saúde Bucal, dos Centros de Especialidades Odontológicas e dos Laboratórios Regionais de Prótese Dentária. 
Os resultados de tais intervenções são perceptíveis quando se analisam os dados das Pesquisas Nacionais de Saúde Bucal demonstrando ter ocorrido melhora considerável nos indicadores; e os dados referentes às ações e serviços de saúde, a partir dos quais se percebe aumento da cobertura populacional atendida.

Medidas de austeridade fiscal, como as tomadas recentemente no Brasil, limitam o orçamento público e fortalecem a saúde suplementar, colocando a PNSB em situação de dificuldades para a sua operacionalização. Diante disto, há iniciativas na área da saúde bucal coletiva para que o Brasil Sorridente se torne uma política do SUS e não apenas restrita a governos, materializada no Projeto de Lei 6836/2017, buscando seu contínuo avanço, com qualidade.

\section{REFERÊNCIAS}

1. Rua MG. Políticas públicas. Florianópolis, Brasil: Departamento de Ciências da Administração, UFSC; 2009.

2. Viana ALA, Baptista TWF. Análise de políticas de saúde. En: Giovanella L, Escorel S, Lobato LVC, Noronha JC, Carvalho AI, organizadores. Políticas e sistemas de saúde no Brasil, (pp. 65-105). Rio de Janeiro, Brasil: Fiocruz; 2008.

3. Serafim MP, Dias RB. Análise de políticas: uma revisão da literatura. Cad gest soc. 2012 jan/jun; 3(1): 121-34.

4. Ham C; Hill M. The policy process in the modern capitalist state. Londres, England: Harvester Wheatsheaf; 1993. 
5. Parsons W. Políticas públicas: una introducción a la teoría y la práctica del análisis de políticas públicas. México, D.F.: Facultad Latinoamericana de Ciencias Sociales e Miño y Dávila Editores, México; 2007.

6. Howlett M; Ramesh M; Perl A. Studying Public Policy. Policy Cycles \& Policy Subsystems. Canadá: Oxford University Press; 2009.

7. Secchi L. Políticas públicas: conceitos, esquemas de análise, casos práticos. 2. Ed. São Paulo, Brasil: Cencage; 2013.

8. Gottems LBD, Pires MRGM, Calmon PCDP, Alves ED. O modelo dos múltiplos fluxos de Kingdon na análise de políticas de saúde: aplicabilidades, contribuições e limites. Saude Soc 2013; 22(2): 511-20.

9. Gil A.C. Métodos e técnicas de pesquisa social. 6.ed. São Paulo, Brasil: Atlas; 2011.

10. Bardin L. Análise de conteúdo. Lisboa, Portugal: Edições 70; 2009.

11. Silva RHA, Sales-Peres A. Odontologia: um breve histórico. Odonto Clín-Cient. 2007; 6(1): $7-11$.

12. Zanetti CHG. As marcas de mal-estar social no sistema nacional de saúde tardio: o caso das políticas de saúde bucal do Brasil nos anos 80. 1993. Dissertação (Mestrado em Saúde Pública) - Escola Nacional de Saúde Pública, Fundação Oswaldo Cruz, Rio de Janeiro, Brasil; 1993.

13. Escorel S, Nascimento DR, Edler FC. As origens da Reforma sanitária e do SUS. En: Lima NT, Gerschman S, Edler FC, Suárez JM, organizadores. Saúde e democracia: história e perspectivas do SUS. Rio de Janeiro, Brasil: Fiocruz; 2005.

14. Paludetto Júnior M. Avaliação de desempenho da política nacional de saúde bucal no período de 2004-2010. 2013. Dissertação (Mestrado em Ciências da Saúde) - Universidade de Brasília, Brasília, Brasil; 2013. 
15. Manfredini MA. Saúde bucal no Brasil em 2008 e nos 20 anos de Sistema Único de Saúde. En: República Federativa do Brasil, Ministério da Saúde. Saúde Brasil 2008: 20 anos de Sistema Único de Saúde (SUS) no Brasil. Brasília, Brasil: Ministério da Saúde; 2009.

16. Narvai PC, Frazão P. Políticas de Saúde Bucal no Brasil. En: Krieger L, Moysés SJ, Moysés ST, editores. Saúde Bucal das Famílias: trabalhando com evidências (pp. 1-20). São Paulo, Brasil: Artes Médicas; 2008.

17. Souza MF. Programa Saúde da Família: estratégia de superação das desigualdades na saúde? Análise do acesso aos serviços básicos de saúde. 2007. Tese (Doutorado em Saúde Pública) Faculdade de Ciências da Saúde da Universidade de Brasília, Brasília; 2007.

18. Roncalli AG. Epidemiologia e saúde bucal coletiva: um caminhar compartilhado. Cienc Saude Colet. 2006; 11(1): 105-14.

19. Krieger L, Moysés SJ, Moysés ST. Saúde Bucal das Famílias: trabalhando com evidências. São Paulo, Brasil: Artes Médicas; 2008.

20. República Federativa do Brasil, Ministério da Saúde. Relatório da $11^{\text {a }}$ Conferência Nacional de Saúde. Brasília, Brasil: Ministério da Saúde; 2010.

21. República Federativa do Brasil, Ministério da Saúde. Portaria GM/MS nº 1.444 de 28 de dezembro de 2000. Estabelece incentivo financeiro para a reorganização da atenção à saúde bucal prestada nos municípios por meio do Programa de Saúde da Família. Brasília, Brasil: Ministério da Saúde; 2000.

22. Machado CV, Baptista TWF, Nogueira CA. Políticas de saúde no Brasil nos anos 2000: a agenda federal de prioridades. Cad. Saúde Pública. 2011; 27(3): 521-32.

23. República Federativa do Brasil, Ministério da Saúde. Portaria 36 de 14 de janeiro de 2004. Brasília, Brasil: Ministério da Saúde; 2004. 
24. Andrade FR. Relações de poder na Política Nacional de Saúde Bucal. Goiânia, Brasil: UFG; 2009.

25. República Federativa do Brasil, Presidência da República. Discurso do Presidente da República, Luiz Inácio Lula da Silva, na cerimônia de lançamento do Programa Nacional de Saúde Bucal em 17 de março de 2004. Brasília, Brasil: Secretaria de Imprensa e Divulgação da Presidência da República, 2004b. Disponível em: http://www.biblioteca.presidencia.gov.br/ex-presidentes/luiz-inacio-lula-dasilva/discursos/1o-mandato/2004/1o-semestre/17-03-2004-discurso-do-presidente-darepublica-luiz-inacio-lula-da-silva-na-cerimonia-de-lancamento-do-programa-nacional-desaude-bucal/download. Acesso em: 3 set. 2018.

26. Fernandes GF. A $3^{\text {a }}$ Conferência Nacional de Saúde Bucal e as políticas públicas de saúde. 2011. Dissertação (Mestrado em Saúde Pública). Faculdade de Saúde Pública, Universidade de São Paulo, São Paulo, Brasil; 2011.

27. Goés PSA, Moysés SJ, organizadores. Planejamento, gestão e avaliação em saúde bucal. Porto Alegre, Brasil: Artes Médicas; 2012.

28. República Federativa do Brasil, Ministério da Saúde. Sala de apoio à gestão estratégica e participativa. Brasília, Brasil: Ministério da Saúde; 2018. Disponível em: http://sage.saude.gov.br. Acesso em: 3 set. 2018.

29. República Federativa do Brasil, Ministério da Saúde. Resultados principais do SB Brasil 2010. Brasília, Brasil: Ministério da Saúde; 2011. 